\title{
Spectral Flow and Maslov Index Arising from Lagrangian Intersections
}

\author{
Nobukazu OTSUKI and Kenro FURUTANI
}

Science University of Tokyo

(Communicated by Y. Shimizu)

Dedicated to Professor Nobuhiko Tatsuuma on his 60-th birthday

\section{§1. Introduction.}

Let $P$ be a $2 n$-dimensional symplectic manifold with the symplectic structure $\omega$, and $L, L^{\prime}$ be two Lagrangian submanifolds of $P$. We assume, in this paper, that $L$ and $L^{\prime}$ intersect transversally with a non-empty intersection, and a smooth map

$$
f: I \times I \rightarrow P, \quad I=[0,1]
$$

is given with the following properties:

$$
\begin{array}{ll}
f(\tau, 0) \in L, & f(\tau, 1) \in L^{\prime} \quad \text { for any } \quad \tau \in I, \\
f(0, t) \equiv x, \quad f(1, t) \equiv y \quad \text { for any } t \in I,
\end{array}
$$

where $x, y \in L \cap L^{\prime}$. Under these assumptions, two homotopic invariants arise. One is the spectral flow associated to a family of certain operators, and the other is the Maslov index of a curve which relates with the boundary conditions imposed on the operators in that family.

A. Floer has shown that these two are equal to relative Morse index from $x$ to $y$, which is defined as the Fredholm index of a certain elliptic operator (see $[F]$ ).

Now let us explain the problem more precisely. Let $g$ be a Riemannian metric on $P$ which is adapted to the symplectic structure $\omega$, that is, if we write $\omega(X, Y)=g(X, J Y)$, for any vector fields $X, Y$ on $P$, then $J$ is an almost complex structure of $P$. Hence $J^{2}=-$ Id and ${ }^{t} J=-J\left({ }^{t} J\right.$ is the transpose of $J$ with respect to the metric $g$ ). We fix such a metric henceforth, and denote by $\nabla$ the Riemannian connection of the metric $g$. Let $\Omega$ be a path space of $P$ consisting of smooth paths $z: I \rightarrow P$ such that $z(0) \in L$ and $z(1) \in L^{\prime}$. Consider, at least locally, a symplectic action functional $a: \Omega \rightarrow R$ defined by 
the equation

$$
\langle d a(z), \xi\rangle=\int_{0}^{1} \omega(\dot{z}, \xi) d t,
$$

where $\xi$ is a smooth section of the induced bundle $z^{*}(T P)$ on $I$, or $\xi$ is a vector field along the curve $\{z(t)\}$, which can be regarded also as a tangent vector at $z$ in $\Omega$. Then we obtain an operator field $\mathscr{A}_{z}$ on $\Omega$ as "Hessian of the functional $a$ ":

$$
\mathscr{A}_{z}(\xi)=J \nabla_{\dot{z}} \xi+\left(\nabla_{\xi} J\right)(\dot{z}), \quad \xi \in C_{0}^{\infty}\left(z^{*}(T P)\right) .
$$

Let $f: I \times I \rightarrow P$ be a map as above, and we denote $\mathscr{A}_{\tau}=\mathscr{A}_{f_{\tau}}$ for the path $f_{\tau}(t)=f(\tau, t)$. We would like to associate two quantities, so-called spectral flow and Maslov index, to the family of operators $\left\{\mathscr{A}_{\tau}\right\}$ both of which depend only on the two points $x, y \in L \cap L^{\prime}$ and the homotopy class of the map $f$. To do so, there are three problems to be made clear:

(1) It can be verified that, if $z \in \Omega$ is a constant path, i.e. $z(t) \equiv x, x \in L \cap L^{\prime}$, then $\mathscr{A}_{z}$ is formally symmetric, or if $P$ is Kähler and $g$ is the Kähler metric, then $\mathscr{A}_{z}$ is symmetric for any path $z \in \Omega$. But the operator $\mathscr{A}_{z}$ is not even formally symmetric in general.

(2) Each operator $\mathscr{A}_{z}$ acts on a different space of sections. We must make the domains of the operators to be identical one, and give a definite meaning of the continuity of the family $\left\{\mathscr{A}_{\tau}\right\}$ with respect to the parameter $\tau$.

(3) The family $\left\{\mathscr{A}_{z}\right\}$ does not form a loop, if $x \neq y$. We would like to make it a loop in a natural way.

Although A. Floer discussed on the coincidence of two quantities in his paper [F] by interceding with a single Fredholm operator ('desuspension' of one parameter family of selfadjoint Fredholm operators (see [APS])), it is not so clear for us about these points (1), (2) and (3). So it is our aim of this paper to make clear these points together with giving a definite description of the two homotopy invariants within the framework given by $[\mathrm{AS}]$, [BW] and [V]. By making clear these points, especially by clarifying the continuity of the family $\left\{\mathscr{A}_{\tau}\right\}$ with respect to the parameter $\tau$, we get a direct proof (without 'desuspending' the family $\left\{\mathscr{A}_{\tau}\right\}$ ) of the coincidence of the two homotopy invariants. Hence we think it is worth while to write this paper.

In $\S 2$, we review the notion "spectral flow" by following [AS] and [BW], and note a slight generalization in the proposition 2.4 , which is used to.define the spectral flow for loops of non-selfadjoint Fredholm operators. Also we explain an example to which our case is reduced in the final step in the proof of the main Theorem 5.1, in $\S 5$.

In §3, we shall construct two loops in Lagrangian-Grassmannian manifold $\Lambda(n)=U(n) / O(n)$ from the data given by the map $f: I \times I \rightarrow P$ with the property (1-1) and show that the Maslov indices of these two loops coincide. One of the two loops is used to construct a loop of operators from the family $\left\{\mathscr{A}_{\tau}\right\}$.

In $\S 4$, we construct a continuous loop in the space of bounded selfadjoint Fredholm operators $\hat{\mathscr{F}}_{*}($ see $\S 2)$ from the family $\left\{\mathscr{A}_{\tau}\right\}$, and in $\S 5$ we show our main Theorem 5.1 . 


\section{§ 2. Spectral flow.}

In this section, we first describe the notion "spectral flow". For more details see [AS], [APS] and [BW].

Let $H_{c}$ be a separable complex Hilbert space of infinite dimension, $\mathscr{F}=\mathscr{F}\left(H_{C}\right)$ the space of bounded Fredholm operators on $H_{c}$, and $\hat{F}$ the subspace of $\mathscr{F}$ consisting of self-adjoint operators.

$\hat{\mathscr{F}}$ has three components $\hat{\mathscr{F}}_{+}, \hat{\mathscr{F}}_{-}$and $\hat{\mathscr{F}}_{*}$ consisting of essentially positive operators, essentially negative operators, and others respectively. It is easy to see that $\hat{\mathscr{F}}_{ \pm}$are contractible.

Define a map

$$
\alpha: \hat{\mathscr{F}}_{*} \rightarrow \Omega \mathscr{F}
$$

by assigning to each $A \in \hat{\mathscr{F}}_{*}$ the path

$$
\cos \pi t+\sqrt{-1} A \sin \pi t \quad \text { for } \quad t \in I,
$$

then we have the following theorem.

THEOREM [AS]. The map $\alpha$ is a homotopy equivalence, and so $\hat{\mathscr{F}}_{*}$ is a classifying space for the functor $K^{-1}$, i.e., for any compact space $X$, we have an isomorphism

$$
K^{-1}(X)=\left[X, \hat{\mathscr{F}}_{*}\right],
$$

where $\left[X, \hat{\mathscr{F}}_{*}\right]$ is the set of homotopy classes of continuous maps from $X$ to $\hat{\mathscr{F}}_{*}$.

From the Bott periodicity theorem and this theorem, we have

$$
\pi_{1}\left(\hat{\mathscr{F}}_{*}\right) \cong Z \text {. }
$$

The isomorphism (2-2) implies that a loop in $\hat{\mathscr{F}}_{*}$ has just a homotopy invariant and it can be characterized by an integer, called, "spectral flow".

We now explain this. Let $\ell: I \rightarrow \mathscr{\mathscr { F }}_{*}$ be a continuous map, and $\ell_{0}$ and $\ell_{1}$ have the same spectral set: $\sigma\left(\ell_{0}\right)=\sigma\left(\ell_{1}\right)$. Then, roughly speaking, the spectral flow of the family $\left\{\ell_{t}\right\}$ is the difference between the number of eigenvalues which change the sign from - to + on $I$ and the number of eigenvalues which change the sign from + to - .

To put it more precisely, let $\hat{F}(\infty)$ be a subspace of $\hat{\mathscr{F}}_{*}$ such that $A \in \hat{F}(\infty)$, if and only if,

the intersection $\sigma(A) \cap(-1,1)$ consists of finite isolated eigenvalues with finite multiplicities, $\sigma_{\text {ess }}(A)=\{-1,1\}$ and the norm of $A$ is equal to $1,\|A\|=1$, where $\sigma_{\text {ess }}(A)$ is the set of essential spectra of the operator $A$ (see [K] for the definitions).

Proposition 2.1 (see [BW]). The space $\hat{F}(\infty)$ is a deformation retract of $\hat{\mathscr{F}}_{*}$.

Let $\ell: I \rightarrow \hat{F}(\infty)$ be a continuous loop $\left(\ell_{0}=\ell_{1}\right.$, and the topology of $\hat{F}(\infty)$ is of 
course the uniform topology). Then the graph of the spectrum of $\ell$ can be parametrized through a finite monotone sequence of continuous functions

$$
\begin{aligned}
\lambda_{j}: & I \rightarrow[-1,1], \quad j=1, \cdots, N, \\
& -1 \leqq \lambda_{1}(t) \leqq \lambda_{2}(t) \leqq \cdots \leqq \lambda_{N-1}(t) \leqq \lambda_{N}(t) \leqq 1 \text { for } t \in I .
\end{aligned}
$$

Then we have

PROPOSITION $2.2[\mathrm{BW}$. Let $\ell: I \rightarrow \hat{F}(\infty)$ be as above, then there exists an integer $s$ such that $\lambda_{k+s}(0)=\lambda_{k}(1)$, for each $k$, where we regard $\lambda_{-|s|}=\lambda_{-|s|+1}=\cdots=\lambda_{0}=-1$, and $\lambda_{N}=\lambda_{N+1}=\cdots=\lambda_{N+|s|}=1$. Moreover the number $s$ is homotopically invariant.

From this proposition we can give the

Definition. For a homotopy class of a loop $\ell: I \rightarrow \hat{F}(\infty)$, we can define $\operatorname{sf}\{\ell\}=s$, where $s$ is an integer in the proposition 2.2 above, and we call it the spectral flow of the loop $\ell$.

Hence by this definition together with the proposition 2.1 we have

PROPOSITION 2.3 ([BW]). The map defined by the spectral flow gives an isomorphism sf : $\pi_{1}\left(\hat{\mathscr{F}}_{*}\right) \cong Z$.

We will now explain some relations between real and complex cases briefly. Let $H_{R}$ be a real Hilbert space, then $\mathscr{F}\left(H_{R}\right), \hat{\mathscr{F}}\left(H_{R}\right), \hat{\mathscr{F}}_{ \pm}\left(H_{R}\right)$ and $\hat{\mathscr{F}}_{*}\left(H_{R}\right)$ are defined in the same ways as for the complex cases, and $\hat{\mathscr{F}}_{*}\left(H_{R}\right)$ is, in this case, a classifying space for the functor $K R^{-7}$ (real $K$-group). Although $\hat{\mathscr{F}}_{*}\left(H_{R}\right)$ and $\hat{\mathscr{F}}_{*}\left(H_{R} \otimes C\right)$ are not homotopic, we have

$$
\pi_{1}\left(\hat{\mathscr{F}}_{*}\left(H_{R}\right)\right) \cong \pi_{1}\left(\hat{\mathscr{F}}_{*}\left(H_{R} \otimes C\right)\right)
$$

through the complexification $\hat{\mathscr{F}}_{*}\left(H_{R}\right) \subset \hat{\mathscr{F}}_{*}\left(H_{R} \otimes C\right)$. This can be done by defining an isomorphism

$$
\text { sf : } \pi_{1}\left(\hat{\mathscr{F}}_{*}\left(H_{R}\right)\right) \cong Z
$$

in the same way as in the complex case.

REMARK 1. In this paper we will work on real Hilbert spaces.

REMARK 2. Let $\hat{F}\left(H_{R}\right)$ be the space of skew-adjoint Fredholm operators on a real Hilbert space $H_{R}$. Various subspaces $\hat{F}^{-k}\left(H_{R}\right)$ of $\hat{F}\left(H_{R}\right)$, for $k=1, \cdots, 7$, are defined through the *-representations of Clifford algebras on $H_{R}$, each of which is a classifying space for the functor $K R^{-k}$, the $k$-th real $K$-group (see [AS]). For example $\hat{F}\left(H_{R}\right)$ itself is a classifying space for $K R^{-1}$. In this case the complexification

$$
\hat{F}\left(H_{R}\right) \subset \hat{F}\left(H_{R} \otimes C\right) \cong \sqrt{-1} \hat{F}_{*}\left(H_{R} \otimes C\right)
$$

induces the trivial map $\pi_{1}\left(\hat{F}\left(H_{R}\right)\right) \cong Z_{2} \rightarrow \pi_{1}\left(\hat{\mathscr{F}}_{*}\left(H_{R} \otimes C\right)\right) \cong Z$. As for the case $k=7$, the 
space $\hat{F}^{-7}\left(H_{\mathbf{R}}\right)$ is isomorphic to the space $\hat{\mathscr{F}}_{*}\left(H_{\boldsymbol{R}}\right)$.

Next we give a proposition.

Proposition 2.4. Let $\mathscr{K}$ be the space of all compact operators on $H(H$ is real or complex), then the inclusion

$$
i: \hat{\mathscr{F}}_{*} \rightarrow \hat{\mathscr{F}}_{*}+\mathscr{K}
$$

is a homotopy equivalence.

Proof. Let $A=S+K, S \in \hat{\mathscr{F}}_{*}, K \in \mathscr{K}$, then $\left(A+A^{*}\right) / 2=S+\left(K+K^{*}\right) / 2 \in \hat{\mathscr{F}}_{*}$. So define a map

$$
j: \hat{\mathscr{F}}_{*}+\mathscr{K} \rightarrow \hat{\mathscr{F}}_{*}
$$

by $j(A)=\left(A+A^{*}\right) / 2$, then $i \circ j$ is homotopic to Id through a homotopy $f_{t}(A)=$ $t A+(1-t) i \circ j(A)$. Because, if $A=S+K, S \in \hat{\mathscr{F}}_{*}, K \in \mathscr{K}$, then

$$
\begin{aligned}
f_{t}(A)= & t(S+K)+(1-t)\left(S+\left(K+K^{*}\right) / 2\right) \\
& =S+((1+t) / 2) K+((1-t) / 2) K^{*} \in \hat{\mathscr{F}}_{*}+\mathscr{K}
\end{aligned}
$$

for each $t \in I$.

Owing to this proposition we have an integer "spectral flow" for a loop $\left\{\ell_{t}\right\}$ in $\hat{\mathscr{F}}_{*}+\mathscr{K}$, which can be regarded as a total number of eigenvalues of $\left\{\ell_{t}\right\}$ across the imaginary axis with directions when $t$ moves from 0 to 1 . Especially we have

COROLlaRY 2.5. If a loop $\ell: I \rightarrow \hat{\mathscr{F}}_{*}+\mathscr{K}$ has the form $\ell_{t}=S_{t}+K_{t}$, where $S: I \rightarrow \hat{\mathscr{F}}_{*}$ and $K: I \rightarrow \mathscr{K}$ are both continuous loops, then $\operatorname{sf}\left\{\ell_{t}\right\}=\operatorname{sf}\left\{S_{t}\right\}$.

Finally we discuss an important example to which our case will be reduced in $\S 5$. Let

$$
J=\left(\begin{array}{cc}
0 & -I_{n} \\
I_{n} & 0
\end{array}\right)
$$

where $I_{n}$ is the $n \times n$ unit matrix, and consider an operator

$$
A=J \frac{d}{d t}: C_{0}^{\infty}(I) \otimes R^{2 n} \rightarrow L_{2}(I) \otimes R^{2 n}
$$

Here function spaces $C_{0}^{\infty}(I)$ and $L_{2}(I)$ (square integrable functions on the interval $I=[0,1])$ are to be considered as those consisting of real valued functions. We will denote $L_{2}(I) \otimes R^{2 n}$ by $H_{R}$ and by $W_{1}$, the first order Sobolev space, i.e., $f \in W_{1}$, if and only if, $f \in H_{R}$ and $f^{\prime} \in H_{R}$ (the norm of $f \in W_{1}$ will be defined as $\|f\|_{1}^{2}=\|f\|^{2}+\left\|f^{\prime}\right\|^{2}$ ).

Let $\left(\boldsymbol{R}^{2 n}, \omega\right)$ be the symplectic vector space with the skew symmetric form $\omega$ defined by $J: \omega(x, y)=(x, J y)$, where $(\cdot, \cdot)$ is the standard Euclidean inner product on $\boldsymbol{R}^{2 n}$.

Henceforth we will use identifications: 


$$
\boldsymbol{R}^{2 n} \cong C^{n} \cong R^{n} \oplus \sqrt{-1} R^{n}
$$

and

$$
S p(n, R) \cap S O(2 n) \cong U(n) .
$$

We also use the decomposition $\boldsymbol{R}^{n} \oplus \sqrt{-1} \boldsymbol{R}^{n} \equiv \lambda_{\mathrm{Re}} \oplus \sqrt{-1} \lambda_{\mathrm{Im}}$. Let $\Lambda(n)$ be the set of all Lagrangian subspaces of $\boldsymbol{R}^{2 n}$ (n-dim subspace on which $\omega$ vanishes). $\lambda_{\mathrm{Re}}$ and $\lambda_{\mathrm{Im}}$ are in $\Lambda(n)$. Under these identifications the unitary group $U(n)$ acts on $\Lambda(n)$ transitively, and we have $\Lambda(n)=U(n) / O(n)\left(O(n)\right.$ is the stationary subgroup of the point $\left.\lambda_{\mathrm{Re}}\right)$.

Let $\lambda$ be a Lagrangian subspace in $R^{2 n}$, and define a subspace of $H_{R}$ as follows:

$$
D_{\lambda}=\left\{f: f \in W_{1}, f(0) \in \lambda_{\text {Re }}, f(1) \in \lambda\right\} \text {. }
$$

Then we have,

Proposition 2.6. The operator $A$ can be extended to $D_{\lambda}$ as a selfadjoint operator. We denote this operator by $A_{\lambda}$.

Next, let $u: I \rightarrow U(n)$ be a $C^{2}$-class curve with $u_{0}=$ Id, and define orthogonal operators $U_{\tau}: H_{R} \rightarrow H_{R}$ by

$$
\left(U_{\tau} f\right)(t)=u_{t \tau}(f(t)) .
$$

Put $\lambda_{\tau}=u_{\tau}\left(\lambda_{\mathrm{Re}}\right)$, and $A_{\tau}=A_{\lambda_{\tau}}$, then we have

Proposition 2.7. The domain of the operator $U_{\tau}^{-1} A_{\tau} U_{\tau}$ is $D_{\lambda_{R o}}$, and

$$
U_{\tau}^{-1} \circ A_{\tau} \circ U_{\tau}=A_{0}+B_{\tau}
$$

where $B_{\tau}$ is given by

$$
\left(B_{\tau} \phi\right)(t)=\left(u_{t \tau}{ }^{-1} \circ J \circ \frac{d}{d t}\left(u_{t \tau}\right)\right)(\phi(t)) .
$$

Moreover $B_{\tau}\left(W_{1}\right) \subset W_{1}$, and $B_{\tau}$ and $U_{\tau}$ are continuous families of operators from $W_{1}$ to $W_{1}$ with respect to the parameter $\tau$ and, of course, also as operators $H_{R} \rightarrow H_{R}$.

Proof. By the definition $\left(U_{\tau} f\right)(0)=f(0)$ and $\left(U_{\tau} f\right)(1)=u_{\tau}(f(1))$. So $D_{\lambda_{\tau}}=U_{\tau}\left(D_{\lambda_{R e}}\right)$. Therefore the domain of $U_{\tau}^{-1} \circ A_{\tau} \circ U_{\tau}$ is $D_{\lambda_{\mathrm{R}}}$. By noting $J \circ u_{\tau}=u_{\tau} \circ J$, a direct calculation gives us the formula (2.3) and (2.4). Since $B_{\tau}$ is a matrix operator, we can easily verify the remainder assertions.

Since the family $\left\{A_{\tau}\right\}$ consists of unbounded operators, we transform each $A_{\tau}$ as follows to obtain a continuous path in $\hat{F}_{*}$ : let

$$
\tilde{A}_{\tau}=A_{\tau} \circ\left(1+A_{\tau}{ }^{2}\right)^{-1 / 2} \text {, }
$$

where we regard that the operator $\left(1+{A_{\tau}}^{2}\right)^{-1 / 2}$ is defined by an integral 


$$
\int_{\Gamma}(1+\lambda)^{-1 / 2}\left(\lambda-A_{\tau}^{2}\right)^{-1} d \lambda,
$$

on a suitably taken contour $\Gamma$ in $C$ (note here that $A_{\tau}$ is a real operator for each $\tau$ ). Then each $\tilde{A}_{\tau}$ is a bounded selfadjoint Fredholm operator on $H_{R}$ with eigenvalues of the form $\lambda_{k}(\tau)\left(1+\lambda_{k}(\tau)^{2}\right)^{-1 / 2}$, where $\lambda_{k}(\tau)$ is the $k$-th eigenvalue of $A_{\tau}$. Then we have

Proposition 2.8. The family $\left\{\tilde{A}_{\tau}\right\}$ is a continuous path in $\hat{\mathscr{F}}_{*}\left(H_{\mathbf{R}}\right)$.

ProOf. Put $T_{\tau}=A_{0}+B_{\tau}$. First recall an inequality

$$
\|f\|_{1} \leqq C\left(\left\|T_{\tau} f\right\|+\|f\|\right)
$$

for any $f \in W_{1} \otimes C$, where the constant $C$ does not depend on the parameter $\tau$.

If we regard the resolvent operator $\left(\lambda-T_{\tau}^{2}\right)^{-1}$ as an operator

$$
\left(\lambda-T_{\tau}^{2}\right)^{-1}: H_{R} \otimes C \rightarrow W_{1} \otimes C,
$$

then by the inequality (2-5) we obtain the following estimate:

$$
\left\|\left(\lambda-T_{\tau}^{2}\right)^{-1}\right\|_{H_{R} \otimes C, W_{1} \otimes C}=O\left(|\lambda|^{-1 / 2}\right),
$$

which is valid uniformly with respect to the parameter $\tau$ on the line $\arg \lambda=$ const $\neq 0$.

For $\tau, \tau^{\prime} \in I$, the differences $T_{\tau}{ }^{2}-T_{\tau^{\prime}}{ }^{2}$ are first order differential operators with $C^{1}$-coefficients and the operator norms as operators from $W_{1}$ to $H_{R}$ satisfy

$$
\left\|T_{\tau}^{2}-T_{\tau^{\prime}}^{2}\right\|_{W_{1}, W_{R}}=O\left(\left|\tau-\tau^{\prime}\right|\right) .
$$

These properties (2-6) and (2-7) together with a resolvent equation

$$
\left(\lambda-T_{\tau}^{2}\right)^{-1}-\left(\lambda-T_{\tau^{\prime}}{ }^{2}\right)^{-1}=\left(\lambda-T_{\tau}^{2}\right)^{-1}\left(T_{\tau^{\prime}}{ }^{2}-T_{\tau}^{2}\right)\left(\lambda-T_{\tau^{\prime}}{ }^{2}\right)^{-1}
$$

give us an estimate of the operator norm from $H_{R}$ to $W_{1}$ :

$$
\left\|\left(\mathrm{Id}+T_{\tau}^{2}\right)^{-1 / 2}-\left(\mathrm{Id}+T_{\tau^{\prime}}{ }^{2}\right)^{-1 / 2}\right\|_{W_{R}, W_{1}}=O\left(\left|\tau-\tau^{\prime}\right|\right) \text {. }
$$

From this estimate (2-8) and the fact that $\left\{T_{\tau}\right\}$ is a continuous family of operators from $W_{1}$ to $H_{R}$, we have the continuity of the family $\left\{T_{\tau} \circ\left(\mathrm{Id}+T_{\tau}{ }^{2}\right)^{-1 / 2}\right\}$ in $\hat{\mathscr{F}}_{*}$ with respect to the parameter $\tau$, and also that of the family $\left\{\tilde{A}_{\tau}\right\}$, since $\tilde{A}_{\tau}=U_{\tau} \circ T_{\tau} \circ\left(\operatorname{Id}+T_{\tau}{ }^{2}\right)^{-1 / 2} \circ$ $U_{\tau}{ }^{-1}$. This completes the proof.

Especially take a path $s: I \rightarrow U(n)$,

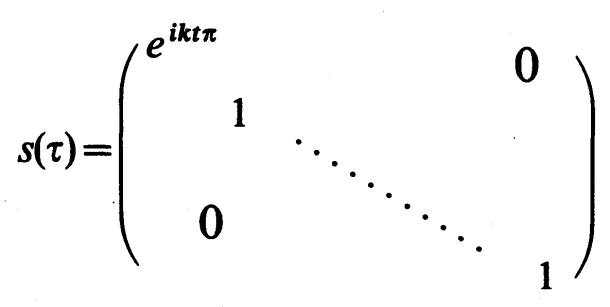


for a fixed integer $k$. Then the path $\left\{\tilde{A}_{\tau}\right\}$ corresponding to the curve $s$ forms a loop in $\hat{\mathscr{F}}_{*}$ and by an explicit calculation of the eigenvalues of this operator $A_{\tau}$ for each $\tau$, we have

Proposition 2.9. The spectral flow of this family is $k: \operatorname{sf}\left\{\tilde{A}_{\tau}\right\}=k$.

ReMARK 3. Let take a $C^{2}$-map $u: I \times I \rightarrow U(n)$ such that $u(0, s)=$ Id for any $s \in I$, instead of the path $u$ in the propositions 2.7 and 2.8 , and denote by $\left\{A_{\tau, s}\right\}$ the selfadjoint extension of the operator $A=J d / d t$ to the domain

$$
D_{\tau, s}=\left\{f \in W_{1}: f(0) \in \lambda_{\mathrm{Re}}, f(1) \in u_{\tau, s}\left(\lambda_{\mathrm{Re}}\right)\right\} .
$$

Then by the same method as in the proof of the proposition 2.8 , the family $\left\{\tilde{A}_{\tau, s}\right\}$, $\tilde{A}_{\tau, s}=A_{\tau, s} \circ\left(\operatorname{Id}+A_{\tau, s}{ }^{2}{ }^{-1 / 2}\right.$, can be shown to be a continuous family of the parameters $\tau$ and $s$. We will use this fact in $\S 5$.

\section{§3. Lagrangian intersection and Maslov index.}

Let $\pi: E \rightarrow X$ be a symplectic vector bundle on a topological space $X$. The structure group of this bundle is $S p(n, R)$, the symplectic group, where $2 n$ is the fiber dimension of $E$, and is reduced to its maximal compact subgroup $U(n)$. This means that $E$ can be regarded as a complex vector bundle on $X$. We denote by $\Lambda(E)$ the LagrangianGrassmannian bundle on $X$ associated to $E$ with the fiber $\Lambda(n)=U(n) / O(n)$.

Put $G(n)=\left\{U \in U(n): \operatorname{det}^{2} U=1\right\}$, then the structure group of $E$ can be reduced to the group $G(n)$, if and only if, $2 c_{1}(E)=0$, where $c_{1}(E)$ is the first Chern class of $E$ as a complex vector bundle. If this condition is satisfied, then there is a map $\alpha: \Lambda(E) \rightarrow U(1)$, which depends on the way of a reduction of the structure group of $E$ from $U(n)$ to $G(n)$. Let $\operatorname{Det}^{2}: \Lambda(n) \rightarrow U(1)$ be the map induced from the map $\operatorname{det}^{2}: U(n) \rightarrow U(1)$, and denote by $[d \theta]$ the generator of $H^{1}(U(1), Z)$, then the class $\left(\operatorname{Det}^{2}\right)^{*}([d \theta])$ is, so called, Keller-Maslov-Arnold characteristic class. If we restrict the class $\alpha^{*}([d \theta])$ to each fiber of $\Lambda(E)$, then it is identified with $\left(\operatorname{Det}^{2}\right)^{*}([d \theta])$.

Also let $\beta: \Lambda(E) \rightarrow U(1)$ be defined by another reduction of the structure group from $U(n)$ to $G(n)$ of the symplectic vector bundle $E$. We have the difference

$$
\alpha^{*}([d \theta])-\beta^{*}([d \theta])=\pi^{*}(c)
$$

with a certain $c \in H^{1}(X, Z)$ (see the appendices of [V]).

In particular, if both $H^{1}(X, Z)$ and $H^{2}(X, Z)$ vanish, then we have a unique class $m \in H^{1}(\Lambda(E), Z), m=\alpha^{*}([d \theta])$, through a map $\alpha: \Lambda(E) \rightarrow U(1)$.

Based on these facts, we now proceed to our problem. Let $(P, \omega)$ be a symplectic manifold of $2 n$-dimension. Let $L$ and $L^{\prime}$ be two Lagrangian submanifolds of $P$, which intersect transversally with a non empty intersection. Then the intersection $L \cap L^{\prime}$ consists of a discrete set of points in $P$. Let points $x$ and $y$ be in $L \cap L^{\prime}$ and a smooth map $f: I \times I \rightarrow P$ satisfy $f(\tau, 0) \in L, f(\tau, 1) \in L^{\prime}, f(0, t) \equiv x$ and $f(1, t) \equiv y$ for all $\tau, t \in I$. 
We take a Riemannian metric $g$ on $P$ which is adapted to the symplectic form $\omega$, and denote by $J$ the almost complex structure defined by $g$ and $\omega$ as in the introduction. Since $T_{x}(L)$ and $J_{x}\left(T_{x}(L)\right)$ are orthogonal, we will use the following identification:

$$
T_{x}(L) \oplus J_{x}\left(T_{x}(L)\right) \equiv R^{n} \oplus \sqrt{-1} R^{n}
$$

and by taking a suitable orthonormal basis in $T_{x}(L)$, we have

$$
J_{x}=\left(\begin{array}{cc}
0 & -I_{n} \\
I_{n} & 0
\end{array}\right)
$$

When two subspaces $\lambda$ and $\mu$ in $\boldsymbol{R}^{2 n}$ are transversal, we will write as follows: $\lambda \Phi \mu$. $t \in I$ :

Let $P_{\tau, t}$ be the parallel displacement along the path $\{f(s, t)\}(0 \leqq s \leqq \tau)$ for each fixed

$$
P_{\tau, t}: T_{x} P \rightarrow T_{f(\tau, t)} P
$$

is an isometry, and put

$$
J_{\tau, t}=P_{\tau, t}^{-1} \circ J_{f(\tau, t)} \circ P_{\tau, t} .
$$

Then we have a smooth map $g: I \times I \rightarrow S O(2 n)$ such that

$$
g_{\tau, t}{ }^{-1} \circ J_{\tau, t} \circ g_{\tau, t}=J_{x} .
$$

Put

$$
\begin{aligned}
& P_{\tau, 0}{ }^{-1}\left(T_{f(\tau, 0)}(L)\right)=\tilde{\lambda}_{0}(\tau), \\
& P_{\tau, 1}{ }^{-1}\left(T_{f(\tau, 1)}\left(L^{\prime}\right)\right)=\tilde{\lambda}_{1}(\tau)
\end{aligned}
$$

and

$$
\begin{aligned}
& g_{\tau, 0}\left(\tilde{\lambda}_{0}(\tau)\right)=\lambda_{0}(\tau), \\
& g_{\tau, 1}\left(\tilde{\lambda}_{1}(\tau)\right)=\lambda_{1}(\tau) .
\end{aligned}
$$

We have the trivialization of $f^{*}(T P)$ by using the parallel displacement $\left\{P_{\tau, t}\right\}$ :

$$
f^{*}(T P) \cong I \times I \times T_{x}(P) \cong I \times I \times R^{2 n}
$$

Then $\lambda_{0}(\tau)$ and $\lambda_{1}(\tau)$ are Lagrangian subspaces in $T_{x}(P) \cong R^{2 n}$, i.e. $\left\{\lambda_{0}(\tau)\right\}$ and $\left\{\lambda_{1}(\tau)\right\}$ are two curves in $\Lambda(n)$. Notice that

$$
\lambda_{0}(0) \Phi \lambda_{1}(0), \quad \lambda_{0}(1) \Phi \lambda_{1}(1) .
$$

There are two ways of constructing loops in $\Lambda(n)$ from these two curves $\left\{\lambda_{0}\right\}$ and $\left\{\lambda_{1}\right\}$, that have the same Maslov index.

We will now explain these ways: Since $\lambda_{0}(0) \Phi \lambda_{1}(0)$ and $\lambda_{0}(1) \Phi \lambda_{1}(1)$, there is a symplectic transformation $\sigma \in S p(n, R)$ such that

$$
\sigma\left(\lambda_{0}(0)\right)=\lambda_{0}(1) \text { and } \sigma\left(\lambda_{1}(0)\right)=\lambda_{1}(1) \text {. }
$$


Take a path $C(\tau)(0 \leqq \tau \leqq 1)$ in $\Lambda(n)$ with

$$
C(0)=\lambda_{0}(0), \quad C(1)=\lambda_{1}(0) .
$$

Then four curves

$$
\lambda_{0}(\tau), \quad \sigma(C(\tau)), \quad \lambda_{1}(1-\tau) \quad \text { and } \quad C(1-\tau) \quad(0 \leqq \tau \leqq 1)
$$

form a loop in $\Lambda(n)$. We denote this loop by $b(s)(0 \leqq s \leqq 4)$. Since the Maslov class $m \in H^{1}(\Lambda(n), Z)$ is invariant under the action of the group $S p(n, R)$ on $\Lambda(n)$, the integer $\int_{b} m$ is independent of the choice of the path $C$.

For constructing another loop, take a curve $U: I \rightarrow U(n)$ such that

$$
U_{t}\left(\lambda_{0}(0)\right)=\lambda_{0}(\tau) \text { and } U_{0}=I d,
$$

where we used the identification (3-1). Put

$$
U_{\tau}^{-1}\left(\lambda_{1}(\tau)\right)=\lambda_{2}(\tau),
$$

then we have

$$
\lambda_{2}(0) \Phi \lambda_{0}(0) \text { and } \lambda_{2}(1) \Phi \lambda_{0}(0) .
$$

So we join $\lambda_{2}(0)$ and $\lambda_{2}(1)$ by a curve $\tilde{C}(\tau)(0 \leqq \tau \leqq 1)$ with the property

$$
\tilde{C}(\tau) \Phi \lambda_{0}(0)
$$

for any $\tau \in I$. Notice that two such curves with the property (3-9) are homotopic to each other. As before, the curves $\tilde{C}(\tau)$ and $\lambda_{2}(1-\tau)(0 \leqq \tau \leqq 1)$ define the loop $\{\tilde{b}(s)\}(0 \leqq s \leqq 2)$. Then we have

Proposition 3.1.

$$
\int_{b} m=\int_{b} m
$$

Proof. Let $H:[0,1] \times[0,4] \rightarrow \Lambda(n)$ be a continuous map such that

$$
H(s, \tau)=\left\{\begin{array}{lll}
U_{s \tau}^{-1}\left(\lambda_{0}(\tau)\right) & 0 \leqq \tau \leqq 1, & 0 \leqq s \leqq 1 \\
U_{s}^{-1}(\sigma(C(\tau-1))) & 1 \leqq \tau \leqq 2, & 0 \leqq s \leqq 1 \\
U_{s(3-\tau)}{ }^{-1}\left(\lambda_{1}(3-\tau)\right) & 2 \leqq \tau \leqq 3, & 0 \leqq s \leqq 1 \\
C(4-\tau) & 3 \leqq \tau \leqq 4, & 0 \leqq s \leqq 1
\end{array}\right.
$$

Then $H$ is a homotopy between the loop $\{b(\tau)\}$ and the loop $\{H(1, \tau)\}$, where $U:[0,1] \rightarrow U(n)$ is the map given in (3-6).

Next, owing to the fact that the group $\operatorname{Sp}(n, R)$ acts transitively on the space of pairs consisting of transversally intersecting Lagrangian subspaces in $R^{2 n}$, we have a map $V: I \rightarrow S p(n, R)$ with the properties: 


$$
\begin{array}{ll}
V_{0}=I d, & \\
V_{\tau}\left(\lambda_{0}(0)\right)=\lambda_{0}(0) & \text { for any } \tau \in I, \\
V_{\tau}\left(\lambda_{2}(0)\right)=\lambda_{2}(\tau) & \text { for any } \tau \in I, \\
V_{1}=U_{1}^{-1} \circ \sigma . &
\end{array}
$$

Note here that, if the map $V_{1}{ }^{-1} \circ U_{1}{ }^{-1} \circ \sigma$ restricted to the subspace $\lambda_{0}(0)$ has determinant -1 , then $\sigma$ should be replaced by another $\tilde{\sigma} \in S p(\mathrm{n}, \boldsymbol{R})$ such that

$$
\tilde{\sigma}\left(\lambda_{0}(0)\right)=\lambda_{0}(1), \quad \tilde{\sigma}\left(\lambda_{1}(0)\right)=\lambda_{1}(1),
$$

and

$$
\operatorname{det}\left(\tilde{\sigma}^{-1} \sigma_{\mid \lambda_{0}(0)}\right)=-1 \text {, }
$$

for the sake that the map $V$ satisfies the property (3-11).

Let $G:[0,1] \times[0,1] \rightarrow \Lambda(n)$ be a continuous map defined by: $G(s, \tau)=V_{s}(C(\tau))$. Then

which implies

$$
\int_{\partial G} m=0
$$

$$
\int_{\tilde{b}} m=\int_{U_{1}-1_{\circ \sigma(c)}} m-\int_{\lambda_{2}} m-\int_{C} m
$$

Hence we have

$$
\int_{b} m=\int_{\tilde{b}} m
$$

REMARK 4. Let $\tilde{b}$ be the above loop. If there exists another Lagrangian subspace $\mu$ satisfying $\mu \Phi \lambda_{2}(\tau)$ for every $\tau \in I$, then the integer $\int_{\tilde{\sigma}} m$ equals the Hörmander index $\sigma\left(\lambda_{0}(0), \mu ; \lambda_{2}(0), \lambda_{2}(1)\right)$ (see $[\mathrm{H}]$ ), because of the very definition of it.

REMARK 5. The following example satisfies the situation in Remark 4: Let $\phi$ be a Morse function on a smooth manifold $M$, the symplectic manifold $P$ be $T^{*}(M)$ in this case, $L=M$, i.e., $M$ is seen as zero sections in $T^{*}(M)$, and $L^{\prime}=d \phi(M)$. Let $x$ and $y$ be in $L \cap L^{\prime}$, that is, $d \phi_{x}=0$ and $d \phi_{y}=0$. Since the vertical foliation of $T^{*}(M)$ is transversal to $d \phi(M)$, we can take $T_{x}\left(T_{x}^{*}(M)\right)$ as the Lagrangian subspace $\mu$ satisfying the above condition in Remark 4. Now let $f: I \times I \rightarrow T^{*}(M)$ be a smooth map satisfying conditions (1-1). Then we have a formula:

$$
\int_{\tilde{b}} m=\operatorname{sign} H_{\phi}(y)-\operatorname{sign} H_{\phi}(x),
$$


where $H_{\phi}(\cdot)$ denotes the Hessian of $\phi$ at critical points. Note that the right hand side of this formula is independent of the map $f$.

\section{§4. Lagrangian intersections and spectral flow.}

Let $(P, \omega), L$ and $L^{\prime}$ be as in $\S 3$. Let $\Omega$ be the path space of $P$ consisting of smooth paths $z: I \rightarrow P$ such that $z(0) \in L$ and $z(1) \in L^{\prime}$. We consider a smooth path $\left\{f_{\tau}\right\}$ in $\Omega$ :

$$
f: I \times I \rightarrow P, \quad f_{\tau}(t) \equiv f(\tau, t) .
$$

Along the path $\left\{f_{\tau}\right\}$, we define a family of operators $\left\{\mathscr{A}_{\tau}\right\}$ as follows:

$$
\mathscr{A}_{\tau}(\xi)(t)=J_{f_{\tau}(t)}\left(\nabla_{f_{\tau}(t)}(\xi)(t)\right)+\left(\nabla_{\xi}\left(J_{f_{\tau}(t)}\right)\right)\left(\dot{f}_{\tau}(t)\right), \quad \xi \in C_{0}^{\infty}\left(f_{\tau}^{*}(T P)\right) .
$$

In the sequel we will construct a loop of operators in $\hat{\mathscr{F}}_{*}$ starting from the family $\left\{\mathscr{A}_{\tau}\right\}$.

Let $\|\cdot\|_{0}$ and $\|\cdot\|_{1}$ be the norms on $C^{\infty}\left(f_{\tau}^{*}(T P)\right)$ defined by

$$
\|\xi\|_{0}^{2}=\int_{0}^{1} g_{f_{\tau}(t)}(\xi(t), \xi(t)) d t
$$

and

$$
\|\xi\|_{1}{ }^{2}=\int_{0}^{1} g_{f_{\tau}(t)}\left(\nabla_{f_{\tau}(t)} \xi(t), \nabla_{f_{\tau}(t)} \xi(t)\right) d t+\|\xi\|_{0}^{2}
$$

respectively. We denote the closure of $C^{\infty}\left(f_{\tau}^{*}(T P)\right)$ with respect to the norms $\|\cdot\|_{0}$ and $\|\cdot\|_{1}$ by $H_{\tau}$ and $W_{\tau}$ respectively. Also let denote by $D_{\tau}$ a subspace of $W_{\tau}$ such that

$$
D_{\tau}=\left\{\xi \in W_{\tau}: \xi(0) \in T_{f(\tau, 0)}(L), \xi(1) \in T_{f(\tau, 1)}\left(L^{\prime}\right)\right\} .
$$

Then we can extend the operator $\mathscr{A}_{\tau}$ to a closed operator in $H_{\tau}$ with the domain $D_{\tau}$, denoting it by $A_{\tau}$.

Henceforth, we first transform operators $A_{\tau}$ without changing the spectral set $\sigma\left(A_{\tau}\right)$. in several steps corresponding to the deformations of the paths in $\S 3$. Consequently we get a family of operators with the same principal part.

Let $P_{\tau}$ be the isomorphism from $H_{0}$ to $H_{\tau}$ defined by

$$
\left(P_{\tau} f\right)(t)=P_{\tau, t}(f(t)), \quad f \in H_{0},
$$

where $P_{\tau, t}$ is the parallel displacement cited in $\S 3$. Put $A_{\tau}^{(\mathbf{1})}=P_{\tau}^{-1} \circ A_{\tau} \circ P_{\tau}$ and $D_{\tau}^{(1)}=P_{\tau}^{-1}\left(D_{\tau}\right)$, then $D_{\tau}^{(1)}$ is the domain of $A_{\tau}^{(1)}$. By recalling

$$
J_{\tau, t}=P_{\tau, t}-1 \circ J_{f(\tau, t)} \circ P_{\tau, t}
$$

we get

$$
A_{\tau}^{(1)}=J_{\tau, t} \frac{d}{d t}+B_{\tau}^{(1)},
$$




$$
D_{\tau}^{(1)}=\left\{\xi \in W_{0}: \xi(0) \in \tilde{\lambda}_{0}(\tau), \xi(1) \in \tilde{\lambda}_{1}(\tau)\right\},
$$

where $B_{\tau}^{(1)}$ is a matrix operator with smooth coefficients with respect to $\tau$ and $t$.

Next, let $g_{\tau}$ be the orthogonal transformations on $H_{0}$ defined by

$$
\left(g_{\tau} f\right)(t)=g(\tau, t)(f(t)), \quad f \in H_{0},
$$

where $g: I \times I \rightarrow S O(2 n)$ is satisfying

$$
g_{\tau, t}{ }^{-1} \circ J_{\tau, t} \circ g_{\tau, t}=J_{x} .
$$

Put $A_{\tau}^{(2)}=g_{\tau}{ }^{-1} \circ A_{\tau}^{(1)} \circ g_{\tau}$ and $D_{\tau}^{(2)}=g_{\tau}{ }^{-1}\left(D_{\tau}^{(1)}\right)$, then $D_{\tau}^{(2)}$ is the domain of the operator $A_{\tau}^{(2)}$ and also we have

$$
\begin{aligned}
& D_{\tau}^{(2)}=\left\{\xi \in W_{0}: \xi(0) \in \lambda_{0}(\tau), \xi(1) \in \lambda_{1}(\tau)\right\} \\
& A_{\tau}^{(2)}=J_{x} \frac{d}{d t}+B_{\tau}^{(2)},
\end{aligned}
$$

where $B_{\tau}^{(2)}$ is a matrix operator with smooth coefficients with respect to $\tau$ and $t$.

Let $U: I \rightarrow U(n)=S p(n, R) \cap S O(2 n)$ be a smooth curve such that $U_{0}=I d$, $U_{\tau}\left(\lambda_{0}(0)\right)=\lambda_{0}(\tau)$ as in $\S 3$. Put $A_{\tau}^{(3)}=U_{\tau}^{-1} \circ A_{\tau}^{(2)} \circ U_{\tau}$ and $D_{\tau}^{(3)}=U_{\tau}^{-1}\left(D_{\tau}^{(2)}\right)$, where $U_{\tau}$ is regarded as an orthogonal transformation on $H_{0}$ defined as usual. Then $D_{\tau}^{(3)}$ is the domain of $A_{\tau}^{(3)}$ and since $U_{\tau}$ and $J_{x}$ commute with each other, we get

$$
\begin{aligned}
& A_{\tau}^{(3)}=J_{x} \frac{d}{d t}+B_{\tau}^{(3)}, \\
& D_{\tau}^{(3)}=\left\{\xi \in W_{0}: \xi(0) \in \lambda_{0}(0), \xi(1) \in \lambda_{2}(\tau)\right\}
\end{aligned}
$$

where $B_{\tau}^{(3)}$ is a matrix operator with smooth coefficients with respect to $\tau$ and $t$.

Let $\tilde{D}_{\tau}^{(3)}$ be a subspace of $W_{0}$ such that

$$
\tilde{D}_{\tau}^{(3)}=\left\{\xi \in W_{0}: \xi(0) \in \lambda_{0}(0), \xi(1) \in \widetilde{C}(\tau)\right\},
$$

where $\tilde{C}(\tau)$ is the (smooth) curve defined in $\S 3$, and denote by $\tilde{A}_{\tau}^{(3)}$ a closed extension of the formal differential operator

$$
\left(P_{\tau} \circ g_{\tau} \circ U_{\tau}\right)^{-1} \circ \mathscr{A}_{\tau} \circ P_{\tau} \circ g_{\tau} \circ U_{\tau}
$$

to the domain $\tilde{D}_{\tau}^{(3)}$. Then, at this stage, we have a loop of unbounded closed operators (not necessarily selfadjoint) corresponding to the loop $\{\tilde{b}(\tau)\}$ in $\Lambda(n)$ (see $\S 3$ ). Let denote this loop of operators by $\left\{\ell_{\tau}\right\}$ :

$$
\ell_{\tau}= \begin{cases}\tilde{A}_{\tau}^{(3)} & 0 \leqq \tau \leqq 1 \\ A_{2-\tau}^{(3)} & 1 \leqq \tau \leqq 2 .\end{cases}
$$

Since the domain of the adjoint operator $\ell_{\tau}^{*}$ is the same with it of $\ell_{\tau}$, the operator $\ell_{\tau}+\ell_{\tau}^{*}$ has the domain of definition: $\widetilde{D}_{\tau}^{(3)}, 0 \leqq \tau \leqq 1$, and $D_{2-\tau}^{(3)}, 1 \leqq \tau \leqq 2$, and is self- 
adjoint. Put $S_{\tau}=1 / 2\left(\ell_{\tau}+\ell_{\tau}^{*}\right)$. Then $S_{\tau}$ has the form

$$
S_{\tau}=T_{\tau}+R_{\tau}
$$

where $T_{\tau}=J_{x} d / d t$ with the domain $\tilde{D}_{\tau}^{(3)}$ for $0 \leqq \tau \leqq 1, D_{2-\tau}^{(3)}$ for $1 \leqq \tau \leqq 2$. The zeroth order term $R_{\tau}$ is a selfadjoint matrix operator with smooth coefficients and forms a continuous family with respect to $\tau$. Finally we have a proposition whose proof can be reduced to the proposition 2.8 .

Proposition 4.1. Put $\tilde{S}_{\tau}=S_{\tau} \circ\left(\operatorname{Id}+S_{\tau}{ }^{2}\right)^{-1 / 2}$, then the family $\left\{\tilde{S}_{\tau}\right\}$ is a continuous loop in $\hat{\mathscr{F}}_{*}$. Moreover $\left\{R_{\tau}\right\}$ is itself a continuous loop of bounded selfadjoint operators.

By means of this proposition and the proposition 2.4, we define the number $\operatorname{sf}\left\{\widetilde{S}_{\tau}\right\}$ as the spectral flow for the family $\left\{\mathscr{A}_{\tau}\right\}$. It should be noted that the definition of $\operatorname{sf}\left\{\mathscr{A}_{\tau}\right\}$ does not depend on the choice of the curve $\{\tilde{C}(\tau)\}$ owing to the remark 3 in $\S 2$.

Remark 6. The Riemannian metric $g$ on $P$ was taken so that it adapts to the symplectic form $\omega$. If we take a Riemannian metric $g$ which satisfies moreover that the spaces $T_{p} L$ and $T_{p} L^{\prime}(p=x, y)$ are orthogonal, then the curve $\left\{\lambda_{2}(\tau)\right\}$ is already a loop, and so is the family $\left\{A_{\imath}\right\}$.

\section{§5. Coincidence of spectral flow and Maslov index.}

At this stage the coincidence of the two quantities is easily shown:

THEOREM 5.1. Let $\left\{\tilde{S}_{\tau}\right\}$ be as above. Then

$$
\operatorname{sf}\left\{\mathscr{A}_{\tau}\right\}=\operatorname{sf}\left\{\tilde{S}_{\tau}\right\}=\int_{\tilde{b}} m .
$$

In particular $\operatorname{sf}\left\{\mathscr{A}_{\tau}\right\}$ is independent of the choice of Riemannian metrics adapted to the sympletic form $\omega$ and is a homotopy invariant of the smooth map $f: I \times I \rightarrow P$ with the properties (1-1).

PROOF. Let $h_{s, \tau}=\left(T_{\tau}+s R_{\tau}\right) \circ\left(\operatorname{Id}+\left(T_{\tau}+s R_{\tau}\right)^{2}\right)^{-1 / 2}(0 \leqq s \leqq 1,0 \leqq \tau \leqq 2)$, then we see the map

$$
\begin{aligned}
h:[0,1] \times[0,2] & \rightarrow \hat{\mathscr{F}}_{*} \\
(s, \tau) & \mapsto h_{s, \tau}
\end{aligned}
$$

is continuous according to the proposition 2.8. Note here that the continuity of the map $h$ can be proved separately on $[0,1] \times[0,1]$ and $[0,1] \times[1,2]$. Hence we have a homotopy between the loop $\left\{\widetilde{S}_{\tau}\right\}$ and $\left\{\widetilde{T}_{\tau}\right\}$, where $\tilde{T}_{\tau}=T_{\tau} \circ\left(\operatorname{Id}+T_{\tau}{ }^{2}\right)^{-1 / 2}$, and $T_{\tau}$ is as follows: 


$$
\begin{array}{lll}
T_{\tau}=J_{x} \frac{d}{d t} \quad \text { on } & \tilde{D}_{\tau}^{(3)} & (0 \leqq \tau \leqq 1), \\
T_{\tau}=J_{x} \frac{d}{d t} \quad \text { on } \quad D_{2-\tau}^{(3)} & (1 \leqq \tau \leqq 2) .
\end{array}
$$

Since the spectral flow is homotopically invariant, it is enough to show that the spectral flow of $\left\{\tilde{T}_{\tau}\right\}$ equals

$$
\int_{\tilde{b}} m
$$

Take a path $d: I \rightarrow U(n)$ such that

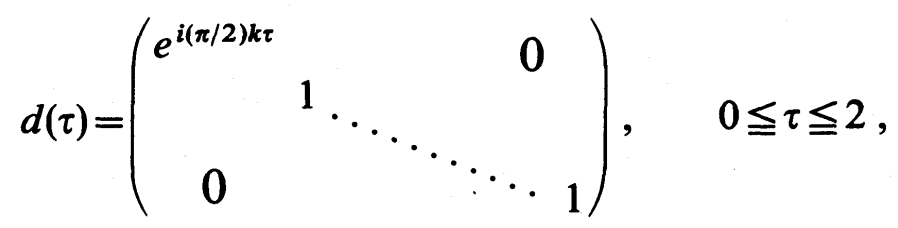

where $k=\int_{b} m$. Then there exist $C^{\infty}$-maps $f^{(0)}$ and $f^{(1)}$

$$
f^{(i)}: I \times I \rightarrow U(n) \quad(i=0,1)
$$

such that for $0 \leqq \tau \leqq 1$

$$
\begin{array}{ll}
f^{(0)}(0, \tau)=d(\tau), & f^{(1)}(0, \tau)=d(1+\tau), \\
f^{(0)}(1, \tau)=\widetilde{C}(\tau), & f^{(1)}(1, \tau)=\lambda_{2}(1-\tau),
\end{array}
$$

and for any $s(0 \leqq s \leqq 1)$

$$
\begin{aligned}
& f^{(0)}(s, 0)=f^{(1)}(s, 0)=\mathrm{Id}, \\
& f^{(0)}(s, 1)\left(\lambda_{0}(0)\right)=f^{(1)}(s, 1)\left(\lambda_{0}(0)\right) .
\end{aligned}
$$

Again, owing to the proposition 2.8 and the remark $3(\S 2)$, we have the loops $\left\{\tilde{T}_{\tau}\right\}$ and $\left\{\tilde{A}_{\tau}\right\}$ (in $\S 2$ ) are homotopic in $\hat{\mathscr{F}}_{*}$. So we have

$$
\operatorname{sf}\left\{\tilde{T}_{\tau}\right\}=\operatorname{sf}\left\{\tilde{A}_{\tau}\right\}=k=\operatorname{sf}\left\{\mathscr{A}_{\tau}\right\}
$$

REMARK 7. Let $T$ be a densely defined closed symmetric operator on a Hilbert space with the same defect indices: $\operatorname{dim} \operatorname{Im}(T-i)^{\perp}=\operatorname{dim} \operatorname{Im}(T+i)^{\perp}=N<+\infty$. If we fix orthonormal basis of $\operatorname{Im}(T-i)^{\perp}$ and $\operatorname{Im}(T+i)^{\perp}$, then selfadjoint extensions of $T$ are parametrized (via Cayley transformation) by $N \times N$ unitary matrices. We denote by $T_{\theta}$ the selfadjoint extension corresponding to a matrix $\theta \in U(N)$.

Now we assume that an extension $T_{\theta_{0}}$ has a compact resolvent (equivalently, all resolvents of any extension are compact), and let $\widetilde{T}_{\theta}=T_{\theta} \circ\left(\mathrm{Id}+T_{\theta}{ }^{2}\right)^{-1 / 2}$. Then we have a map 


$$
\varepsilon: U(N) \rightarrow \hat{\mathscr{F}}, \quad \varepsilon(\theta)=\tilde{T}_{\theta} .
$$

In this paper, we have shown, for the case $T=J d / d t$ (here, $N=2 n$ and we regard that it is complexified), that some curves in $U(2 n)$ with a suitable differentiability condition are mapped by $\varepsilon$ to continuous curves in $\hat{\mathscr{F}}_{*}$.

Whereas the spectrum of $T_{\theta}$ changes 'continuously', when $\theta$ moves continuously (see $[\mathrm{K}]$ ) and also the image of the map $\varepsilon$ is contained in a component of $\hat{\mathscr{F}}$, it seems that the map $\varepsilon$ itself will not be continuous in general. We are interested in what case the map $\varepsilon$ is continuous and how the map $\varepsilon_{*}: \pi_{1}(U(N)) \rightarrow \pi_{1}(\hat{\mathscr{F}})$ is characterized.

\section{References}

[AS] M. F. AtiYAh and I. M. Singer, Index theory for skew-adjoint Fredholm operators, Inst. Hautes Etudes Sci. Publ. Math., 37 (1969), 5-26.

[APS] M. F. AtiYAh, V. K. PATOdi and I. M. Singer, Spectral asymmetry and Riemannian geometry III, Math. Proc. Cambridge Philos. Soc., 79 (1976), 71-99.

[BW] B. Booss and K. WoJCIECHOwski, Desuspension of Splitting Elliptic Symbols, Imfufa Tekst, 52 (1982).

[F] A. Floer, A relative Morse index for the symplectic action, Comm. Pure Appl. Math., 41 (1988), $393-407$.

[FO] K. FURUTANI and N. Otsuki, A space of generalized Atiyah-Patodi-Singer boundary conditions and a formula of a spectral flow, in preparation.

[H] L. HöRmander, Fourier integral operators I, Acta Math., 127 (1971), 79-183.

[K] T. KATO, Perturbation Theory for Linear Operators, 2nd ed., Springer-Verlag, 1976.

[V] C. VITERBo, Intersection de sous-variétés Lagrangiennes, fonctionnelles d'action et indice des systèmes Hamiltoniens, Bull. Soc. Math. France, 115 (1987), 361-390.

Present Address:

Department of Mathematics, Faculty of Science and Technology

SCIENCE UNIVERSITY OF TOKYO

NodA, CHIBA 278, JAPAN 